\title{
Advanced Graphical User Interface for Analysis of Infrared Thermographic Sequence using MATLAB
}

\author{
M. Kante ${ }^{1 *}$, D. Reddy ${ }^{2}$ \\ ${ }^{1}$ Narayana Engineering College, Nellore, India \\ ${ }^{2}$ Andhra University College of Engineering, Vishakaptanam, India \\ \{murali.kante@gmail.com\}
}

\begin{abstract}
The Infrared non-destructive evaluation (IRNDE) is an emerging approach for non-contact inspection of various solid materials such as metals, composites and semiconductors for industrial and research interest. Data processing is an essential step in IRNDE in order to visualize the subsurface defects in the sample and determine the shapes and sizes of the same. The data processing intends to analyse temporal variations in the contrast of each pixel, relative to defect-free reference point on the sample. For that, several post processing algorithms are applied to the recorded thermograms. In this work, it is proposed to an advanced graphical user interface (GUI) for processing the recorded thermograms by IR camera using MATLAB, that supports preprocessing, processing and quantification is presented. Investigations arecarried out using the thermal image sequence recorded with different active thermographic methods: frequency modulated thermal wave imaging (FMTWI) Pulse thermography (PT), lock in thermography (LT). A comparative analysis of the results from different themographic techniques for defect visualization is presented.
\end{abstract}

Keywords: Graphical user interface (GUI), image processing, active thermography

\section{Introduction}

During the past few years, the infrared thermography has been the promising technique to locate subsurface defects in inspected subjects. Based on the method of excitation, it is classified in to several categories viz. ultrasonic imaging, radiographic, eddy current, acoustic emission, heat energy (Infrared), vibration etc. Infrared thermography has become a more popular nondestructive inspection method to evaluate subsurface defects in metallic, insulating, and composite materials, by virtue of its fast inspection rate, noncontact, portability, and easy interpretation. The raw images (thermograms) obtained from IR camera do not often give exact information about defects. The analysis of the raw data is essential to find discontinuities and defects present on the analyzed specimen. This analysis is mainly divided into three parts: pre-processing, processing and analysis of results. This paper is focused on study of different processing techniques to thermographic sequences (thermograms)recorded with different active thermographic methods: frequency modulated thermal wave imaging (FMTWI)[1], Pulse thermography (PT)[2], lock in thermography (LT)[3] . A Graphical user interface (GUI) is developed, that is suitable for both qualitative and quantitative analysis of thermographic sequences. The main goal of the GUI is to cover the evaluation needs of researchers to assess the sample. In this paper, a Graphical interface that supports preprocessing, processing and quantification is presented. Additionally, this GUI shall help researchers to better understand the strengths and weaknesses of the existing algorithms. The GUI is intended to loading images from data base, display them and perform their processing.

\section{Thermal Image}

A thermographic sequence is composed of several hundreds of thermograms. The sample under test is irradiated using a source of infrared radiation, and then the infrared thermal camera records transmitted /reflected radiation. The recorded thermal image provides information about the thermo-physical properties, defects and inhomogenities inside the sample. Thermal mages obtained from experiments always have high noise, especially for metal materials due to high reflectivity and environmental factors, which also need to be processed.

B. Iyer, S. Nalbalwar and R. Pawade (Eds.)

ICCASP/ICMMD-2016. Advances in Intelligent Systems Research.

Vol. 137, Pp. 187-193.

(C) 2017. The authors - Published by Atlantis Press

This is an open access article under the CC BY-NC license (http://creativecommons.org/licens)es/by-nc/4.0/).

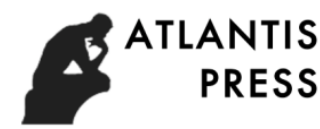




\section{Related Work}

In the literature, we can find many existing packages of this type that are available for processing thermograms such as the open-source IR View Toolbox [4]., the Altair-Li suite provided by Cedip, RTools by FLIR [ 5, 6]., the ThermoFitPro software by Innovation Inc.,[7] and Termidge[8].Although several methods have been proposed to investigate defects, flaws, voids etc in materials/systems, none has so far been free from certain disadvantages. The main objective of this GUI is ensure loading thermal images from data base, display them and perform their preprocessing, processing and quantification to characterize the possible subsurface failures inside sample

\section{Materials and Experimentation}

Experimentation has been carried over mild steel containing 24 drilled bottom holes of diameter $1 \mathrm{~cm}$ each kept at different depths from the non defective end. A frequency modulated chirped stimulation of frequencies swept from $0.01 \mathrm{~Hz}$ to $0.1 \mathrm{~Hz}$ in $100 \mathrm{~s}$, has been provided to the sample with the help of two halogen lamps of power $1 \mathrm{~kW}$ each.

\section{Graphical User Interface}

In order to present the results of the research in an accessible platform a Graphical User Interface (GUI) has been designed. The tool is comprehensive in that it is capable of performing different analysis as required by the user. The tool is coded using Matlab Version 12.The Graphical User Interface enables the user to have seamless use and flexibility of operation. The implementation is carried out in a system having Core 2 Duo processor cloaking at a speed of $2 \mathrm{GHz}$ with a RAM of 2GB.The Structure of the GUI is given in the figure (1)

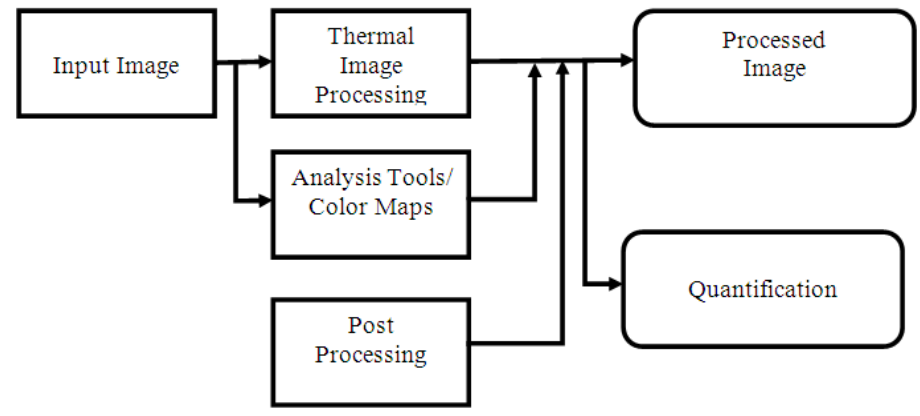

Fig. 1. Basic Structure of the GUI

Screenshot of the GUI designed is illustrated in the Figure (2). The tool as such can be demarcated into 6 different functional regions. Each region has specific functions and analysis elements inbuilt in to them.

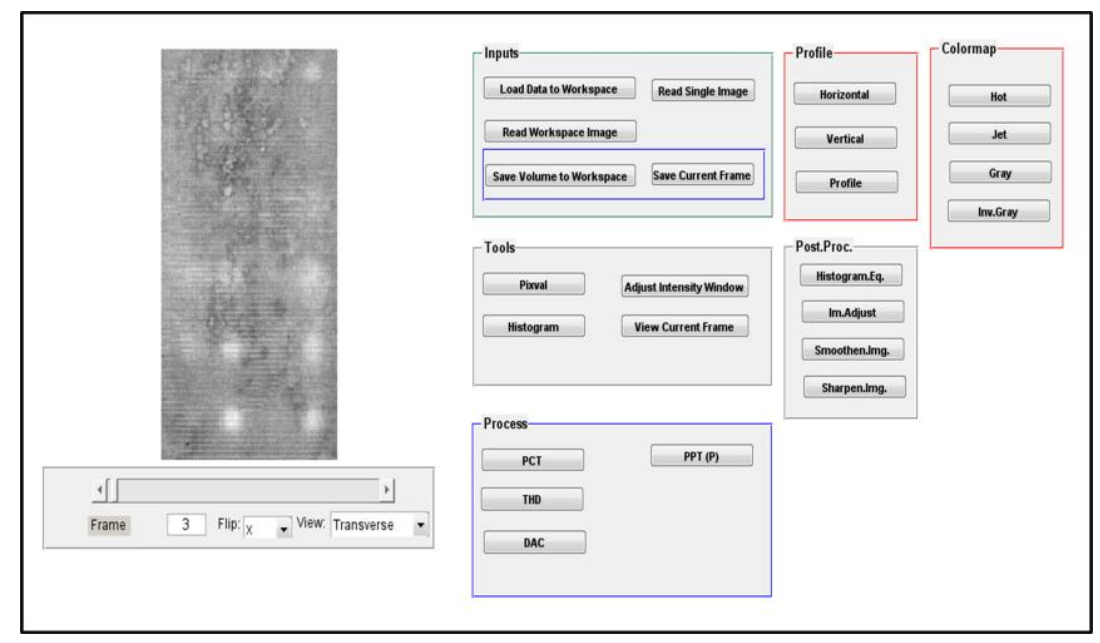

Fig. 2. Screen shot of the GUI 


\subsection{Section 1}

This is the Input / Output section of the tool. The tool is capable of handling images in normal image formats like, .jpg, .bmp, .etc... Images and images that are layered in the Volume format. Most of the image data for thermal analysis are usually in the form of volume images as series of image frames.

This section has the necessary functions to load the image data in to workspace and further read the work space volume for further processing. In the output section, function element enable to save the image in current display window for further analysis. Similarly the current volume can be saved for further processing and analysis.

\subsection{Section 2}

This section helps the user in performing basic analysis of the images. This section has 3 sub functional sections like; Histogram Analysis, Pixel Profile of a particular region of the image, a specific tool for adjusting the image intensity for better visualization and a specific function to visualize the current frame.

The histogram profile of the images serves to give a trend in distribution of intensity values and help in the initial stages of the choosing the threshold. The histogram of the pre and post thermal processing are illustrated in the figure (3)
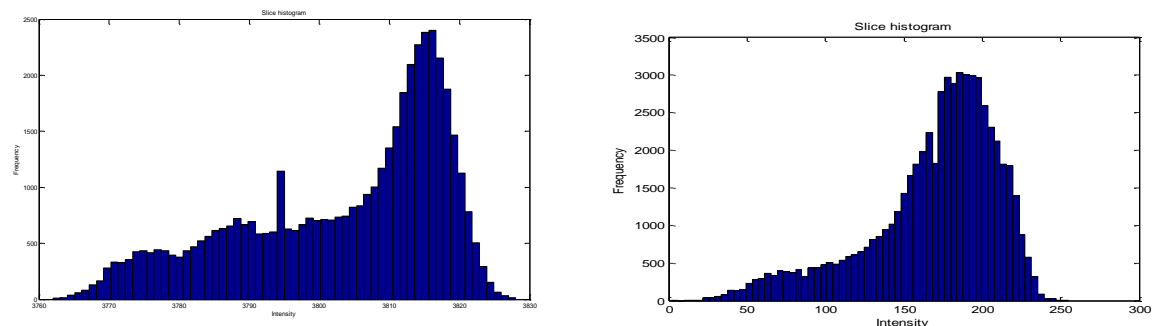

Fig. 3. Histogram profile of an specific slice before and after processing.

From figure (3) it can be clearly inferred that there is an appreciable shift in the histogram of the image before and after thermal processing. Histogram analysis gives a crucial indication of the representation of the pixels which inturn can help in the visulisation of the Processing.

Manual visual interpretation and analysis also play a very critical role in the evlaution procedure, the adjust intensity range tool helps in manual adjustement of the threshold value there by providing better visualisation. The ouput of the adjust inetnsity function is illustrated in the figure (4)

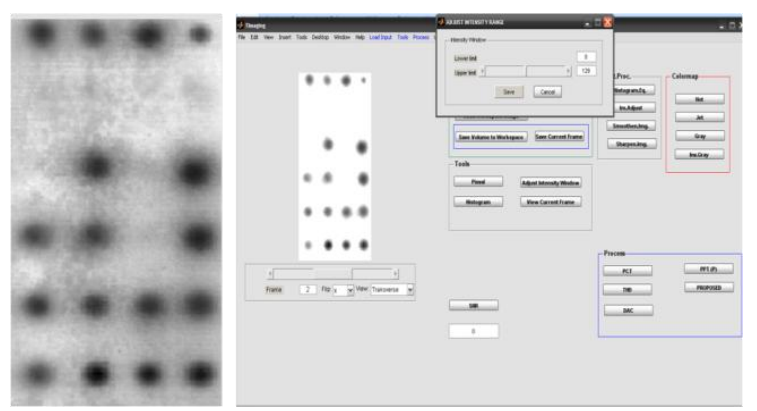

Fig. 4. Resultant output adjusts intensity function in GUI

Similarly other functions of this section include identification of pixel value at a particular pointer position and facility to visualize an individual frame.

\subsection{Section 3}

This section helps the user in performing further analysis of the images. This section has sub functional sections like; Histogram Equalization, image adjusting, smoothening sharpening functions along with different color maps. 
Intensity profile anlysis is another usefull indication about the qaulity of segmentation. Also intensity profile gives the user an idea about distribution of pixel, post processing and pre processing. The intensity profile of a particualr region can also give an inclination towards presence of faults. The intensity profile of processed image across a particular row is given for illustaration in the following figure (5) and figure (6).
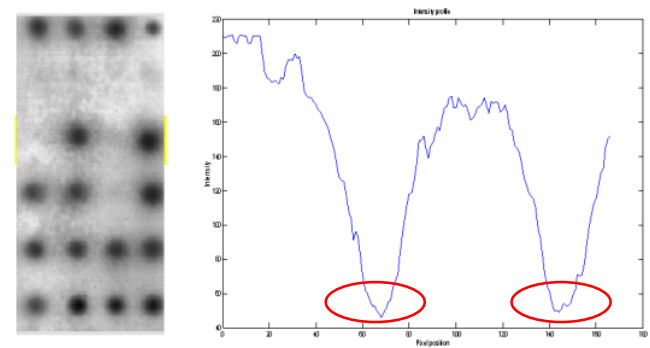

Fig. 5. Intensity Profile of the particular row (top) marked in the image
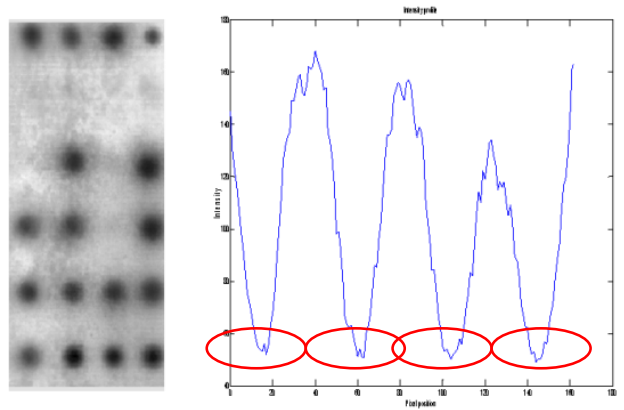

Fig. 6. Intensity Profile of the particular row (bottom) marked in the image

From the figure (5) and figure (6) it can be clearly observed that there is clear difference in pixel profiles between the two rows selected. There is a clear indication in intensity whenever there is a fault and in the test images considered in this work they are holes. This difference in intensity values can give a clear indication of the holes and can help in identification and quantization. The troughs indicate the presence of holes, it can be observed from figure (5) 3 troughs corresponds to the presence of 2 clearly identified holes and in figure (6) there are 4 troughs corresponding to 4 holes in the processed image.

Post processing in processed image plays a crucial role in critical analysis of processed image. Since most of image acquisition process results in very few frames that can be used for analysis and interpretation it is imperative to have post processing techniques that can help in proper analysis of existing information. In this tool 4 functions in the form of im.adjust, Histogram Equalization, Smoothening and Sharpening have been implemented. The results of these operations on processed image are illustrated in the figure (7).

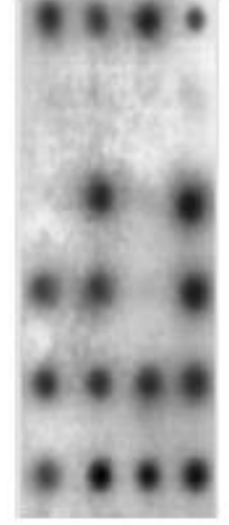

(a)

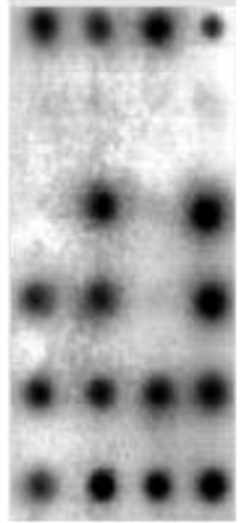

(b)

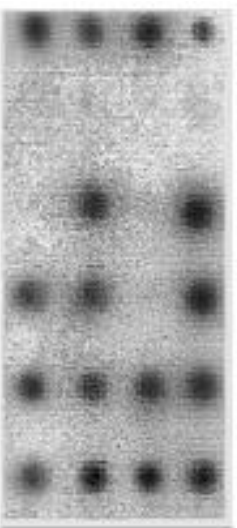

(c)

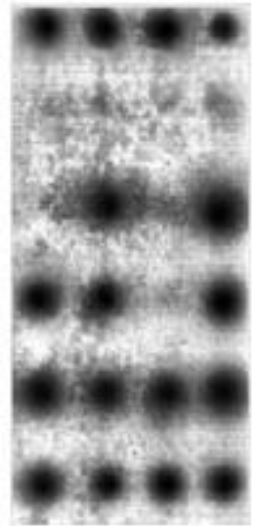

(d)

Fig. 7. Results of operation a) Histogram Equalization, b) imdjust, c) Smoothening, and d)Sharpening. 
Colormap enables the option of viewing the image in different color spaces like, hot, gray and jet. This helps in better visualization of images. The human brain perceives changes in the lightness parameter as changes in the data much better than, for example, changes in hue. Therefore, colormaps which have monotonically increasing lightness through the colormap will be better interpreted by the viewer. Different color space images representation is presented in the figure (8)

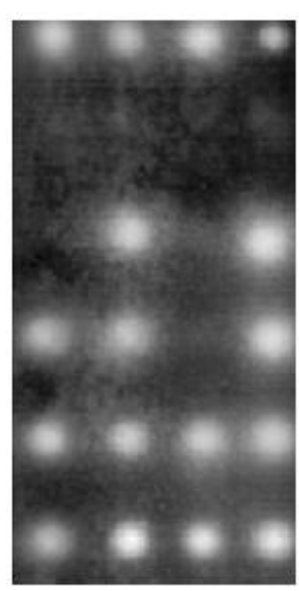

(a)

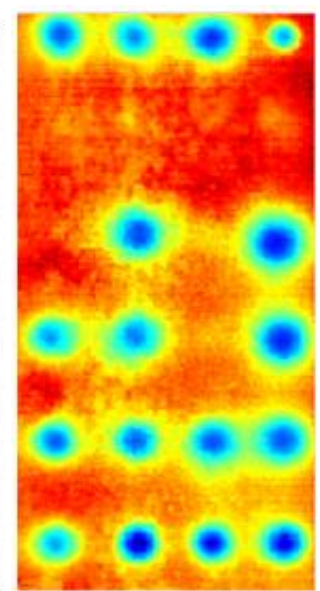

(b)

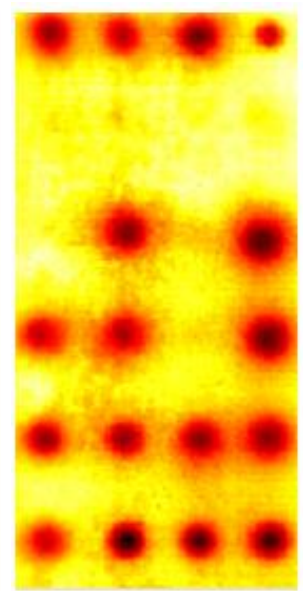

(c)

Fig. 8. An Image represented in different color maps of hot, jet and inverse gray.

\subsection{Section 4}

This section has functions which implement the different thermal processing approaches. In this section we have incorporated, Principal Component Thermography (PCT), Total Harmonic Distortion (DHT), Differential Absolute Contrast (DAC), Pulse Phase Thermography (PPT) and a new method which is proposed based on improved PCT. While choosing the particular method for analysis, user will be asked to input the frame numbers to start and end the processing. This feature will be of great help in analyzing huge volume of data where the user can analyze segment by segment.

Since the scope of the journal is to elaborate on the tool designed, the methods are not explained here. The results of different processing methods are illustrated in the following figure (9).

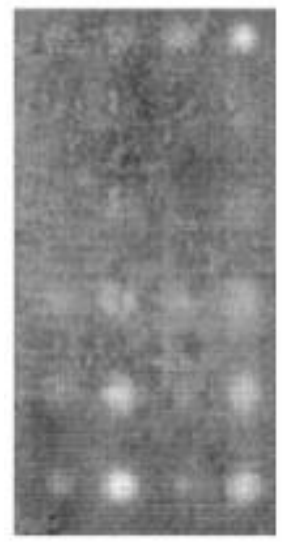

(a)

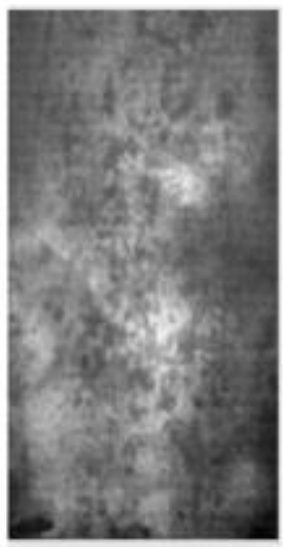

(b)

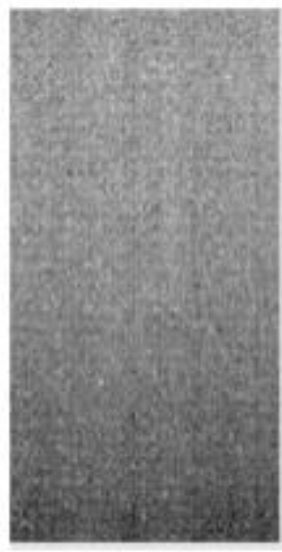

(c)

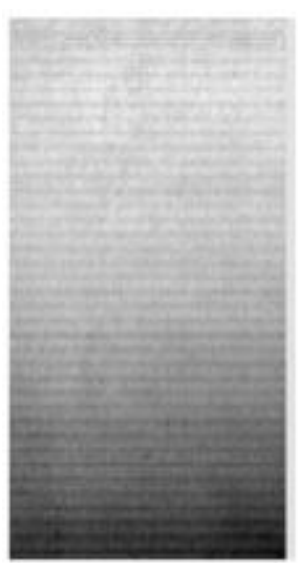

(d)

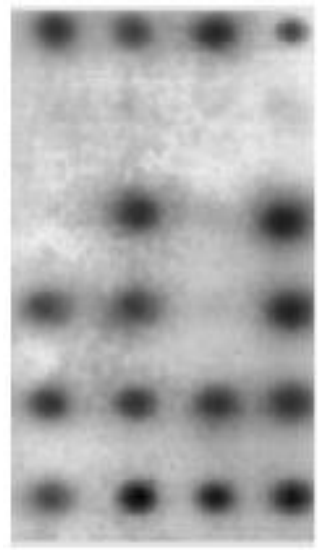

(e)

Fig. 9. Results of thermal image processing by a) PCT, b)THD, c)DAC, d)PPT and e)Proposed Method

The THD method among other things also produces a plot of different parameters like SNR, amplitude of harmonic distortion etc. This is illustrated in the figure (10). 


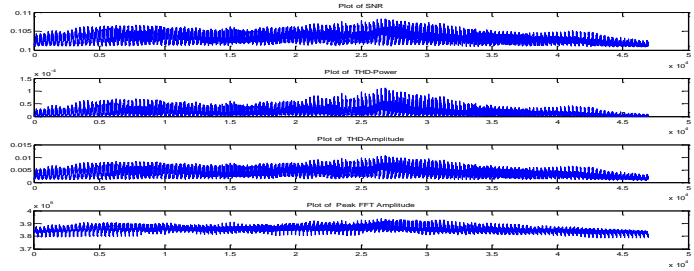

Fig. 10. Plots of different parameters produced by THD

\subsection{Section 5}

This section displays the results of different operations and analysis performed with the help of different function available in the tool box. It has options to choose and view a particular frame in the display section.

\subsection{Section 6}

The quantifiable results are displayed in this section. For example the SNR of a particular point in the image can be visualized here. The SNR changes with depth and can be useful tool in analyzing the quality of thermal processing as well.

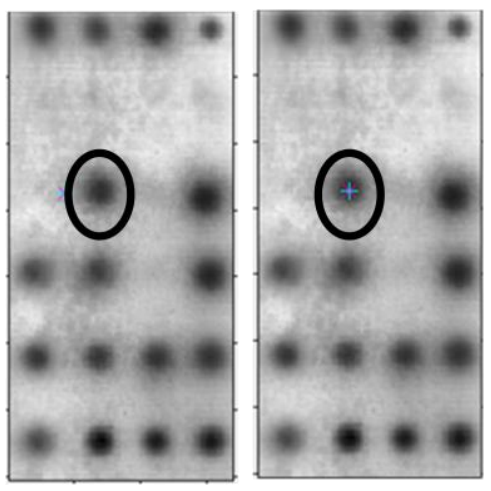

Fig. 11. Points Selected for Analyzing SNR

The above figure (11) illustrates the selections of points for analyzing the SNR .SNR at point adjacent to hole was identified as 83.72 and the SNR at the centre of the hole was identified at 71.26

\section{Conclusions}

Here, a comprehensive framework is presented help researchers in the domain of thermal image processing. The prime objective behind this endeavor is to design a frame tool that has the ease of use and versatility in adding thermal image analysis with specific emphasis on identification of faults. The proposed tool is capable of providing a seamless and flexible user experience and also is capable of providing multi modal analysis. The tool is modular in that it can easily accommodate other analysis procedures as well. It is concluded that, the proposed would be very helpful to researchers for carrying out tasks such as error analysis, system comparison and graphical representations

\section{References}

[1] R. Mulaveesala and S. Tuli, ,'Applications Frequency modulated thermal wave imaging for nondestructive characterization, NCTP'07,American Institute of Physics, 15. 2007

[2] S.M. Shepard ," Advances in Pulsed Thermography" Proceedings of SPIE Vol. 4360, 511. 2001

[3] Datong Wu, Cerd Busse, , "Lock -in thermography for nondestructive evaluation of materials", Rev. Gin. Therm. 37, 693, 1998 
[4] M. Klein, C. Ibarra-Castanedo, X. Maldague, and A. H. Bendada, VIR-View: a straightforward graphical user interface for basic and advanced signal processing of thermographic infrared sequences," in Thermosense XXX, SPIE Defense and Security Symposium, V. P. Vavilov and D. D. Burleigh, Eds., vol. 6939, p. 693914, Orlando, Florida, USA, Vol. 6939, March 16-20 2008.

[5] R. Jones and S. Pittb, using Altair-li in "An experimental evaluation of crack face energy dissipation," International Journal of Fatigue Volume 28, Issue 12, December 2006, Pages 1716-1724, 2006,

[6] F. Escourbiac, S. Constans, X. Courtois, A. Durocher, using Altair-li in "Application of lock-in thermography non destructive technique to CFC armoured plasma facing components", Journal of Nuclear Materials 367-370 1492-1496, 2007

[7] Software ThermoFitPro, from Innovation Inc. http://www.innovation.tomsk.ru/ind_en.html

[8] Vavilov V.P, Kourtenkov D., Grinzato E., Bison P., Marinetti S., Bressan C., Inversion of Experimental Data andThermal Tomography Using „Thermo Heat” and „Termidge” Software, Proc. QIRT'94, p. 273-278, 1994. 